Celina Rom (Bielsko-Biała)

\title{
CONDITIONAL DIFFERENTIAL EQUATIONS
}

Abstract. We introduce and study conditional differential equations, a kind of random differential equations. We give necessary and sufficient conditions for the existence of a solution of such an equation. We apply our main result to a Malthus type model.

1. Introduction. In this paper we introduce the definition of conditional differential equations, a random version of ordinary differential equations. We obtain necessary and sufficient conditions for the existence of a solution of such equations. We can use them to describe various phenomena. We give an example of an application of our results to a model of Malthusian growth of a population size. Results that we obtain using conditional differential equations are similar to those obtained by using more advanced methods [1], 2], [4].

2. Preliminaries. We use the following notation throughout our paper. Let $\mathbb{R}_{+}$denote the set of positive real numbers and let $\mathbb{N}$ denote the set of positive integers. For $n \in \mathbb{N}$ and real numbers $a_{1}, \ldots, a_{n}, b_{1}, \ldots, b_{n}$ such that $a_{i}<b_{i}$ for $i=1, \ldots, n$, the set $K=\left[a_{1}, b_{1}\right] \times \cdots \times\left[a_{n}, b_{n}\right]$ is called a cuboid. We write $\mathbf{x}=\left(x_{1}, \ldots, x_{n}\right)$ for vectors in $\mathbb{R}^{n}$. For $n \in \mathbb{N}, \mathbf{x}, \mathbf{y} \in \mathbb{R}^{n}$, we denote by $\|\mathbf{x}\|_{\mathbb{R}^{n}},\|(\mathbf{x}, \mathbf{y})\|_{\mathbb{R}^{2 n}}$ the usual norms of $\mathbf{x}$ and $(\mathbf{x}, \mathbf{y})$ in $\mathbb{R}^{n}$ and $\mathbb{R}^{2 n}$ respectively. For a fixed $n \in \mathbb{N}$, we denote by $\mathcal{L}_{n}$ the family of Lebesgue measurable sets included in $\mathbb{R}^{n}$, and by $\mu$ the Lebesgue measure defined on $\mathcal{L}_{n}$.

2010 Mathematics Subject Classification: 34A12, 34B60, 35A09, 92D25.

Key words and phrases: ordinary differential equations, Liouville equation, random variable, conditional differential equations.

Received 17 May 2015; revised 28 January 2016.

Published online 18 April 2016. 
3. Conditional differential equations. Now we are going to define conditional differential equations which will be considered in this paper.

Fix $a, b \in \mathbb{R}$ and $n \in \mathbb{N}$, and suppose $0 \in(a, b)$. Let

$$
\mathcal{D}=\{J=(\alpha, \beta): \alpha, \beta \in[a, b] \text { and } \alpha<\beta\} .
$$

For $J \in \mathcal{D}$, let $G_{J}$ denote the set of all functions $\omega: J \rightarrow \mathbb{R}^{n}$ which have continuous first and second derivatives on $J$ and satisfy

$$
\begin{array}{ll}
\lim _{t \rightarrow \alpha^{+}}\left\|\left(\omega(t), \omega^{\prime}(t)\right)\right\|_{\mathbb{R}^{2 n}=\infty} & \text { if } \alpha \neq a, \\
\lim _{t \rightarrow \beta^{-}}\left\|\left(\omega(t), \omega^{\prime}(t)\right)\right\|_{\mathbb{R}^{2 n}=\infty} & \text { if } \beta \neq b .
\end{array}
$$

Throughout this paper, we consider the sets

$$
\Omega=\bigcup_{J \in \mathcal{D}} G_{J} \quad \text { and } \quad \Omega_{t}=\bigcup_{\{J \in \mathcal{D}: t \in J\}}\{\omega \in \Omega: \operatorname{dom}(w)=J\} \quad \text { for } t \in(a, b) .
$$

Let $A \in \mathcal{L}_{2 n}$ and $t, t_{1} \in(a, b)$. We define

$$
A_{t}=\left\{\omega \in \Omega:\left(\omega(t), \omega^{\prime}(t)\right) \in A\right\} \quad \text { and } \quad A_{t, t_{1}}=\Omega_{t} \cap A_{t_{1}} .
$$

Let $\Sigma$ denote the $\sigma$-field generated by the family of sets $\left\{A_{t}: A \in \mathcal{L}_{2 n}\right.$, $t \in(a, b)\}$. Let $\Sigma_{t}$ denote the $\sigma$-field generated by the family of sets $\left\{A_{t, t_{1}}\right.$ : $\left.t_{1} \in(a, b), A \in \mathcal{L}_{2 n}\right\}$.

Notice that $\Sigma_{t} \subset \Sigma$ for every $t \in(a, b)$. Let $\nu$ be a measure on the measurable space $(\Omega, \Sigma)$. Suppose that $\nu\left(\Omega_{t}\right)=1$ for every $t \in(a, b)$. Given such a measure $\nu$ we define random vectors $X_{t}, Y_{t}, W_{t, \Delta t}$ on the space $\left(\Omega_{t}, \Sigma_{t}, \nu\right)$ in the following way:

$$
\begin{aligned}
& X_{t}=\left(X_{1 t}, \ldots, X_{n t}\right), \quad X_{i t}(\omega)=\omega_{i}(t) \quad \text { for } \omega \in \Omega_{t}, i=1, \ldots, n, \\
& Y_{t}=\left(Y_{1 t}, \ldots, Y_{n t}\right), \quad Y_{i t}(\omega)=\omega_{i}^{\prime}(t) \quad \text { for } \omega \in \Omega_{t}, i=1, \ldots, n \text {, }
\end{aligned}
$$

and

$W_{t, \Delta t}=\left(X_{1 t}+\Delta t Y_{1 t}, \ldots, X_{n t}+\Delta t Y_{n t}\right)=\left(W_{1, t, \Delta t}, \ldots, W_{n, t, \Delta t}\right), \quad \Delta t \in \mathbb{R}$.

Let $\mathrm{M}_{0}$ be the family of measures $\nu$ on $(\Omega, \Sigma)$ satisfying the following conditions:

(a) $\nu\left(\Omega_{t}\right)=1$ for any $t \in(a, b)$,

(b) the vector $\left(X_{1 t}, \ldots, X_{n t}\right)$ has the joint probability density function $\varrho_{X_{t}}$ continuous in $\mathbb{R}^{n}$, for any $t \in(a, b)$,

(c) the vector $\left(X_{1 t}, \ldots, X_{n t}, Y_{1 t}, \ldots, Y_{n t}\right)$ has the joint probability density function $\varrho_{X_{t}, Y_{t}}$ continuous in $\mathbb{R}^{2 n}$, for any $t \in(a, b)$,

(d) for any $t \in(a, b)$ and $\Delta t \in \mathbb{R}$, the vector $\left(W_{1, t, \Delta t}, \ldots, W_{n, t, \Delta t}\right)$ has the joint probability density function $\varrho_{W_{t, \Delta t}}$ continuous in $\mathbb{R}^{n}$. 
For a fixed $\nu \in \mathrm{M}_{0}$, we define

$$
\begin{aligned}
c: \mathbb{R}^{n} \times(a, b) \rightarrow \mathbb{R}, & c(\mathbf{x}, t) & =\varrho_{X_{t}}(\mathbf{x}), \\
d: \mathbb{R}^{n} \times \mathbb{R}^{n} \times(a, b) \rightarrow \mathbb{R}, & d(\mathbf{x}, \mathbf{y}, t) & =\varrho_{X_{t}, Y_{t}}(\mathbf{x}, \mathbf{y}), \\
w: \mathbb{R}^{n} \times(a, b) \times \mathbb{R} \rightarrow \mathbb{R}_{+}, & w(\mathbf{x}, t, \Delta t) & =\varrho_{W_{t, \Delta t}}(\mathbf{x}),
\end{aligned}
$$

for $\mathbf{x}, \mathbf{y} \in \mathbb{R}^{n}, t \in(a, b)$ and $\Delta t \in \mathbb{R}$. Notice that the functions $c, d, w$ are uniquely determined by the measure $\nu$ because $\nu$ determines the density functions $\varrho_{X_{t}}, \varrho_{X_{t}, Y_{t}}, \varrho_{W_{t, \Delta t}}$, for each $t \in(a, b)$.

Let $\mathrm{M}$ denote the subset of $\mathrm{M}_{0}$ consisting of all $\nu \in \mathrm{M}_{0}$ such that

(1a) $c(\mathbf{x}, t)>0$ for all $\mathbf{x} \in \mathbb{R}^{n}$ and $t \in(a, b)$,

(2a) $c$ is of class $C^{1}$ on $\mathbb{R}^{n} \times(a, b)$,

(3a) there exists $a \in \mathbb{R}_{+}$such that $c(\mathbf{x}, t) \leq a, \frac{\partial c}{\partial t}(\mathbf{x}, t) \leq a$ and $\frac{\partial c}{\partial x_{i}}(\mathbf{x}, t)$ $\leq a$ for $i=1, \ldots, n$ and $(\mathbf{x}, t) \in \mathbb{R}^{n} \times(a, b)$,

(4a) $\lim _{\Delta t \rightarrow 0} \frac{c(\mathbf{x}, t+\Delta t)-w(\mathbf{x}, t, \Delta t)}{\Delta t}=0$ for all $\mathbf{x} \in \mathbb{R}^{n}$ and $t \in(a, b)$.

For each fixed $\nu \in \mathrm{M}, t \in(a, b)$ and $\mathbf{x} \in \mathbb{R}^{n}$, we denote by $f_{Y_{t}}\left(\mathbf{y} \mid X_{t}=\mathbf{x}\right)$ the conditional probability density function of $Y_{t}$ given $X_{t}=\mathbf{x}$.

Let $p: \mathbb{R}^{n} \times \mathbb{R}^{n} \times(a, b) \rightarrow \mathbb{R}$ satisfy:

(1b) $p(\mathbf{y}, \mathbf{x}, t)>0$ for all $\mathbf{x}, \mathbf{y} \in \mathbb{R}^{n}$ and $t \in(a, b)$,

(2b) $\int_{\mathbb{R}^{n}} p(\mathbf{y}, \mathbf{x}, t) d \mu(\mathbf{y})=1$ for all $\mathbf{x} \in \mathbb{R}^{n}$ and $t \in(a, b)$.

Let $\varrho_{0}: \mathbb{R}^{n} \rightarrow \mathbb{R}$ satisfy:

(1c) $\varrho_{0}(\mathbf{x})>0$ for any $\mathbf{x} \in \mathbb{R}^{n}$,

(2c) $\int_{\mathbb{R}^{n}} \varrho_{0}(\mathbf{x}) d \mu(\mathbf{x})=1$,

(3c) $\varrho_{0}$ is of class $C^{1}$ on $\mathbb{R}^{n}$,

(4c) there exists $a \in \mathbb{R}_{+}$such that $\varrho_{0}(\mathbf{x})<a$ and $\frac{\partial \varrho_{0}}{\partial x_{i}}(\mathbf{x})<a$ for all $\mathbf{x} \in \mathbb{R}^{n}$ and $i=1, \ldots, n$.

Our aim is to find a measure $\nu \in \mathrm{M}$ such that for all $\mathbf{x}, \mathbf{y} \in \mathbb{R}^{n}$ and $t \in(a, b)$, for every $\mathbf{x} \in \mathbb{R}^{n}$.

Equation (Ia) is called a conditional differential equation with the initial condition (Ib). Each measure $\nu \in \mathrm{M}$ for which conditions (Ia) and (Ib) are fulfilled is called a solution of (Ia) with the initial condition (Ib).

The solution measure $\nu$ may not be a probability measure on the whole space $(\Omega, \Sigma)$, but $\nu\left(\Omega_{t}\right)=1$ for all $t \in(a, b)$. Therefore $\nu$ defines a probability measure $P_{t}$ on $\left(\Omega_{t}, \Sigma_{t}\right)$ for each fixed $t \in(a, b)$.

In the remaining part of our paper we additionally assume that

(3b) $p$ is of class $C^{2}$ on $\mathbb{R}^{n} \times \mathbb{R}^{n} \times(a, b)$, 
(4b) for every fixed cuboid $K \subset \mathbb{R}^{n}$ and $t \in(a, b)$, there exist a sufficiently small closed interval $L$ and an integrable function $g$ : $\mathbb{R}^{n} \rightarrow \mathbb{R}_{+}$such that $0 \in L, \int_{\mathbb{R}^{n}} g d \mu(\mathbf{y})<\infty$, and for all $\mathbf{x} \in K$, $\Delta t \in L, \mathbf{y} \in \mathbb{R}^{n}, i=1, \ldots, n$, we have

(i) $\left|y_{i} p(\mathbf{y}, \mathbf{x}-\Delta t \mathbf{y}, t)\right| \leq g(\mathbf{y})$,

(ii) $\left|y_{i} \frac{\partial p}{\partial x_{i}}(\mathbf{y}, \mathbf{x}-\Delta t \mathbf{y}, t)\right| \leq g(\mathbf{y})$.

For $i=1, \ldots, n$, define $E_{i}: \mathbb{R}^{n} \times(a, b) \rightarrow \mathbb{R}$ by

$$
E_{i}(\mathbf{x}, t)=\int_{\mathbb{R}^{n}} y_{i} p(\mathbf{y}, \mathbf{x}, t) d \mu(\mathbf{y}) \quad \text { for } \mathbf{x} \in \mathbb{R}^{n} \text { and } t \in(a, b) .
$$

Notice that it follows from (4b) that $\left|E_{i}(\mathbf{x}, t)\right|<\infty$ for all $\mathbf{x} \in \mathbb{R}^{n}, t \in(a, b)$ and $i=1, \ldots, n$. Moreover,

(5b) each $E_{i}$ is of class $C^{1}$ on $\mathbb{R}^{n} \times(a, b)$.

4. Main result. We give a necessary condition for the existence of a solution of some conditional equation.

Theorem 1. Let $p: \mathbb{R}^{n} \times \mathbb{R}^{n} \times(a, b) \rightarrow \mathbb{R}$ satisfy conditions $(1 \mathrm{~b})-(5 \mathrm{~b})$, and let $\varrho_{0}: \mathbb{R}^{n} \rightarrow \mathbb{R}$ satisfy conditions (1c)-(4c). Assume that the conditional differential equation (Ia) with the initial condition (Ib) has a solution $\nu \in \mathrm{M}$ (so conditions $(\mathrm{a})-(\mathrm{d})$ and (1a)-(4a) are fulfilled). Then the functions $c$ and $E_{i}, i=1, \ldots, n$, determined by the measure $\nu$ as in Section 3 satisfy the equation

(Ic) $\frac{\partial c}{\partial t}+\sum_{i=1}^{n} \frac{\partial\left(c E_{i}\right)}{\partial x_{i}}=0 \quad$ with the initial condition $c(\mathbf{x}, 0)=\varrho_{0}(\mathbf{x})$,

where $\mathbf{x} \in \mathbb{R}^{n}$ and $t \in(a, b)$.

Proof. Let $c, d$ and $w$ be defined from $\nu$ (as in Section 3). Using known formulas for the density function of a random variable we obtain

$$
w(\mathbf{x}, t, \Delta t)=\int_{\mathbb{R}^{n}} d(\mathbf{x}-\Delta t \mathbf{y}, \mathbf{y}, t) d \mu(\mathbf{y})
$$

for $\mathbf{x} \in \mathbb{R}^{n}$ and $t, t+\Delta t \in(a, b)$. Since $\nu \in M$ condition (4a) is satisfied. We will show that under our assumptions this condition is equivalent to (Ic). For $\mathbf{x} \in \mathbb{R}^{n}$ and $t, t+\Delta t \in(a, b)$, we have

$$
c(\mathbf{x}, t+\Delta t)-w(\mathbf{x}, t, \Delta t)=c(\mathbf{x}, t+\Delta t)-\int_{\mathbb{R}^{n}} d(\mathbf{x}-\Delta t \mathbf{y}, \mathbf{y}, t) d \mu(\mathbf{y}) .
$$


By the Taylor formula we obtain

$$
\begin{aligned}
c(\mathbf{x}, t+\Delta t) & -w(\mathbf{x}, t, \Delta t) \\
= & c(\mathbf{x}, t)+\frac{\partial c}{\partial t}(\mathbf{x}, t) \Delta t+\left(\frac{\partial c}{\partial t}\left(\mathbf{x}, t+\theta_{1, \mathbf{x}}\right)-\frac{\partial c}{\partial t}(\mathbf{x}, t)\right) \Delta t \\
& -\int_{\mathbb{R}^{n}}\left(d(\mathbf{x}, \mathbf{y}, t)+\sum_{i=1}^{n} \frac{\partial d}{\partial x_{i}}(\mathbf{x}, \mathbf{y}, t)\left(-y_{i} \Delta t\right)\right) d \mu(\mathbf{y}) \\
& -\int_{\mathbb{R}^{n}}\left(\sum_{i=1}^{n}\left(\frac{\partial d}{\partial x_{i}}\left(\mathbf{x}-\theta_{2, \mathbf{y}} \mathbf{y}, \mathbf{y}, t\right)-\frac{\partial d}{\partial x_{i}}(\mathbf{x}, \mathbf{y}, t)\right)\left(-y_{i} \Delta t\right)\right) d \mu(\mathbf{y}),
\end{aligned}
$$

where $\theta_{1, \mathbf{x}}, \theta_{2, \mathbf{y}}$ are between 0 and $\Delta t$. Hence

$$
\begin{aligned}
& c(\mathbf{x}, t+\Delta t)-w(\mathbf{x}, t, \Delta t)=c(\mathbf{x}, t)+\frac{\partial c}{\partial t}(\mathbf{x}, t) \Delta t \\
& \quad+\left(\frac{\partial c}{\partial t}\left(\mathbf{x}, t+\theta_{1, \mathbf{x}}\right)-\frac{\partial c}{\partial t}(\mathbf{x}, t)\right) \Delta t-c(\mathbf{x}, t)+\sum_{i=1}^{n} \int_{\mathbb{R}^{n}} y_{i} \frac{\partial d}{\partial x_{i}}(\mathbf{x}, \mathbf{y}, t) \Delta t d \mu(\mathbf{y}) \\
& \quad+\sum_{i=1}^{n} \int_{\mathbb{R}^{n}} y_{i}\left(\frac{\partial d}{\partial x_{i}}\left(\mathbf{x}-\theta_{2, \mathbf{y}} \mathbf{y}, \mathbf{y}, t\right)-\frac{\partial d}{\partial x_{i}}(\mathbf{x}, \mathbf{y}, t)\right) \Delta t d \mu(\mathbf{y}) .
\end{aligned}
$$

From condition $(2 \mathrm{a})$, we obtain

$$
\lim _{\Delta t \rightarrow 0} \frac{\left(\frac{\partial c}{\partial t}\left(\mathbf{x}, t+\theta_{1, \mathbf{x}}\right)-\frac{\partial c}{\partial t}(\mathbf{x}, t)\right) \Delta t}{\Delta t}=0 .
$$

Moreover, using conditions (2a), (3a), (3b), (4b) and taking into account that $d(\mathbf{x}, \mathbf{y}, t)=c(\mathbf{x}, t) p(\mathbf{y}, \mathbf{x}, t)$, we get

$$
\begin{aligned}
\lim _{\Delta t \rightarrow 0} \int_{\mathbb{R}^{n}} \frac{y_{i}\left(\frac{\partial d}{\partial x_{i}}\left(\mathbf{x}-\theta_{2, \mathbf{y}} \mathbf{y}, \mathbf{y}, t\right)-\frac{\partial d}{\partial x_{i}}(\mathbf{x}, \mathbf{y}, t)\right) \Delta t}{\Delta t} d \mu(\mathbf{y}) \\
=\int_{\mathbb{R}^{n}} \lim _{\Delta t \rightarrow 0}\left(y_{i}\left(\frac{\partial d}{\partial x_{i}}\left(\mathbf{x}-\theta_{2, \mathbf{y}} \mathbf{y}, \mathbf{y}, t\right)-\frac{\partial d}{\partial x_{i}}(\mathbf{x}, \mathbf{y}, t)\right)\right) d \mu(\mathbf{y})=0
\end{aligned}
$$

for $i=1, \ldots, n$. Thus

$$
\lim _{\Delta t \rightarrow 0} \frac{c(\mathbf{x}, t+\Delta t)-w(\mathbf{x}, t, \Delta t)}{\Delta t}=\frac{\partial c}{\partial t}(\mathbf{x}, t)+\sum_{i=1}^{n} \int_{\mathbb{R}^{n}} y_{i} \frac{\partial d}{\partial x_{i}}(\mathbf{x}, \mathbf{y}, t) d \mu(\mathbf{y}) .
$$

Now, using conditions (2a), (3a), (3b), (4b), (5b) and again taking into account that $d(\mathbf{x}, \mathbf{y}, t)=c(\mathbf{x}, t) p(\mathbf{y}, \mathbf{x}, t)$, we get

$$
\begin{aligned}
\frac{\partial c}{\partial t}(\mathbf{x}, t)+\sum_{i=1}^{n} \int_{\mathbb{R}^{n}} y_{i} \frac{\partial d}{\partial x_{i}}(\mathbf{x}, \mathbf{y}, t) d \mu(\mathbf{y}) & \\
= & \frac{\partial c}{\partial t}(\mathbf{x}, t)+\sum_{i=1}^{n} \frac{\partial}{\partial x_{i}}\left(\int_{\mathbb{R}^{n}} y_{i} d(\mathbf{x}, \mathbf{y}, t) d \mu(\mathbf{y})\right)
\end{aligned}
$$




$$
\begin{aligned}
& =\frac{\partial c}{\partial t}(\mathbf{x}, t)+\sum_{i=1}^{n} \frac{\partial}{\partial x_{i}}\left(\int_{\mathbb{R}^{n}} y_{i} c(\mathbf{x}, t) p(\mathbf{y}, \mathbf{x}, t) d \mu(\mathbf{y})\right) \\
& =\frac{\partial c}{\partial t}(\mathbf{x}, t)+\sum_{i=1}^{n} \frac{\partial}{\partial x_{i}}\left(c(\mathbf{x}, t) \int_{\mathbb{R}^{n}} y_{i} p(\mathbf{y}, \mathbf{x}, t) d \mu(\mathbf{y})\right) \\
& =\frac{\partial c}{\partial t}(\mathbf{x}, t)+\sum_{i=1}^{n} \frac{\partial}{\partial x_{i}}\left(c(\mathbf{x}, t) E_{i}(\mathbf{x}, t)\right) .
\end{aligned}
$$

Theorem 1 is proved.

The next theorem gives sufficient conditions for the existence of a solution of a conditional differential equation.

THEOREM 2. Suppose that the functions $p=p(\mathbf{y}, \mathbf{x}, t)$ and $E_{i}=E_{i}(\mathbf{x}, t)$ satisfy conditions $(1 \mathrm{~b})-(5 \mathrm{~b})$. Suppose that $\varrho_{0}=\varrho_{0}(\mathbf{x}), \mathbf{x} \in \mathbb{R}^{n}$, satisfies conditions (1c)-(4c). Moreover, suppose that (Ic) has exactly one solution $c=c(\mathbf{x}, t)$ for $(\mathbf{x}, t) \in \mathbb{R}^{n} \times(a, b)$. Assume that the function $c$ satisfies conditions $(1 \mathrm{a})-(3 \mathrm{a})$, and additionally:

(5a) $\int_{\mathbb{R}^{n}} c(\mathbf{x}, t) d \mu(\mathbf{x})=1$ for all $t \in(a, b)$,

(6a) $c$ is of class $C^{2}$ in $\mathbb{R}^{n} \times(a, b)$.

Then the conditional differential equation (Ia) with the initial condition (Ib) has a solution $\nu \in \mathrm{M}$.

In the proof of Theorem 2, we construct a measure $\nu \in \mathrm{M}$ which is a solution of the conditional differential equation. Our proof is based on 3 , Theorem 2] and on the following lemma.

LEMMA 1. Under the notation and assumptions of Theorem 2 , let $d=$ $d(\mathbf{x}, \mathbf{y}, t)=p(\mathbf{y}, \mathbf{x}, t) c(\mathbf{x}, t)$ for $\mathbf{x}, \mathbf{y} \in \mathbb{R}^{n}$ and $t \in(a, b)$. Then there exist functions $F_{1}, \ldots, F_{n}: \mathbb{R}^{n} \times \mathbb{R}^{n} \times(a, b) \rightarrow \mathbb{R}$ such that $F_{1}, \ldots, F_{n-1}$ are of class $C^{2}, F_{n}$ is of class $C^{1}$ on $\mathbb{R}^{n} \times \mathbb{R}^{n} \times(a, b)$ and

$$
\frac{\partial d}{\partial t}+\sum_{i=1}^{n} \frac{\partial}{\partial x_{i}}\left(d y_{i}\right)+\sum_{i=1}^{n} \frac{\partial}{\partial y_{i}}\left(d F_{i}\right)=0
$$

for all $\mathbf{x}, \mathbf{y} \in \mathbb{R}^{n}$ and $t \in(a, b)$.

Proof. Fix any functions $F_{1}, \ldots, F_{n-1}: \mathbb{R}^{n} \times \mathbb{R}^{n} \times(a, b) \rightarrow \mathbb{R}$ of class $C^{2}$. Our aim is to construct a function $F_{n}$ such that $F_{1}, \ldots, F_{n}$ satisfy the assertion of Lemma 1. Let us consider equation (IIc) with our functions $F_{1}, \ldots, F_{n-1}$ as parameters and the unknown function $F_{n}$. It follows from (IIc) that 


$$
\begin{aligned}
F_{n}(\mathbf{x}, \mathbf{y}, t)= & -\frac{1}{d}\left(\int_{0}^{y_{n}}\left(\frac{\partial d}{\partial t}(\mathbf{u})+\sum_{i=1}^{n} \frac{\partial\left(d y_{i}\right)}{\partial x_{i}}(\mathbf{u})+\sum_{i=1}^{n-1} \frac{\partial\left(d F_{i}\right)}{\partial y_{i}}(\mathbf{u})\right) d \xi\right) \\
& +\frac{1}{d} \eta\left(x_{1}, \ldots, x_{n}, y_{1}, \ldots, y_{n-1}, t\right),
\end{aligned}
$$

where $\mathbf{u}=\left(x_{1}, \ldots, x_{n}, y_{1}, \ldots, y_{n-1}, \xi, t\right)$ for fixed $\mathbf{x}, \mathbf{y} \in \mathbb{R}^{n}, t \in(a, b)$ and $\eta: \mathbb{R}^{2 n-1} \times(a, b) \rightarrow \mathbb{R}$ is a fixed function of class $C^{1}$. The assumptions on $F_{1}, \ldots, F_{n-1}$ and $p, c, \eta$ guarantee that $F_{n}$ is of class $C^{1}$. Moreover, $F_{1}, \ldots, F_{n}$ satisfy (IIc).

Proof of Theorem 2. We are looking for a measure $\nu \in \mathrm{M}$ such that

$$
\varrho_{X_{t}, Y_{t}}(\mathbf{x}, \mathbf{y})=d(\mathbf{x}, \mathbf{y}, t)=p(\mathbf{y}, \mathbf{x}, t) c(\mathbf{x}, t) \quad \text { for } t \in(a, b) \text { and } \mathbf{x}, \mathbf{y} \in \mathbb{R}^{n} .
$$

Let $F_{1}, \ldots, F_{n}: \mathbb{R}^{n} \times \mathbb{R}^{n} \times(a, b) \rightarrow \mathbb{R}$ be as in Lemma 1 . In particular all these functions are of class $C^{1}$ and satisfy (IIc) for $\mathbf{x}, \mathbf{y} \in \mathbb{R}^{n}$ and $t \in(a, b)$. Then the function $d$ is a local integral invariant of the system of differential equations

$$
x_{1}^{\prime}=y_{1}, \ldots, x_{n}^{\prime}=y_{n}, \quad y_{1}^{\prime}=F_{1}, \ldots, y_{n}^{\prime}=F_{n}
$$

(cf. [3, Theorem 2]). Denote by $\mathrm{C}$ the set of all saturated solutions of (Id) (i.e. solutions which have no non-trivial extension). Notice that for $\widetilde{\omega} \in \mathrm{C}$, we have $\widetilde{\omega}(t)=\left(\omega(t), \omega^{\prime}(t)\right)$ for any $\omega \in \Omega, \omega: J \rightarrow \mathbb{R}^{n}$ and $t \in J$. Let

$$
\overline{\mathrm{C}}=\{\omega \in \Omega: \widetilde{\omega} \in \mathrm{C}\} .
$$

For $t_{0} \in(a, b)$ and $\mathbf{x}_{0}, \mathbf{y}_{0} \in \mathbb{R}^{n}$, we denote by $\widetilde{\omega}_{\mathbf{x}_{0}, \mathbf{y}_{0}, t_{0}}$ the function $\widetilde{\omega} \in \mathrm{C}$ such that $\widetilde{\omega}\left(t_{0}\right)=\left(\mathbf{x}_{0}, \mathbf{y}_{0}\right)$. Let $A \in \mathcal{L}_{2 n}$. We denote

$$
(A)_{t}=\{\omega \in \Omega: \widetilde{\omega} \in \mathrm{C}, \widetilde{\omega}(t) \in A\} .
$$

Let

$$
\mathrm{D}=\left\{(A)_{t}: A \in \mathcal{L}_{2 n}, t \in(a, b)\right\} \cup\{\Omega \backslash \bar{C}\} .
$$

Define $\rho: \mathrm{D} \rightarrow \mathbb{R}$ by

$$
\rho\left((A)_{t}\right)=\int_{A} d(\mathbf{x}, \mathbf{y}, t) d \mu(\mathbf{x}, \mathbf{y}) \quad \text { and } \quad \rho(\Omega \backslash \bar{C})=0 .
$$

Notice that $\rho\left(\left(A_{1}\right)_{t_{1}}\right)=\rho\left(\left(A_{2}\right)_{t_{2}}\right)$ for $A_{1}, A_{2} \in \mathcal{L}_{2 n}$ and $t_{1}, t_{2} \in(a, b)$ such that $\left(A_{1}\right)_{t_{1}}=\left(A_{2}\right)_{t_{2}}$ (cf. [3, Theorem 1]). So, our definition is correct.

Let $\mathrm{E} \subset \Omega$ and $S=\left\{\bigcup_{k \in \mathbb{N}}\left(A_{k}\right)_{t_{k}}:\left(A_{k}\right)_{t_{k}} \in \mathrm{D}, k \in \mathbb{N}, \mathrm{E} \subset \bigcup_{k \in \mathbb{N}}\left(A_{k}\right)_{t_{k}}\right\}$. We define $\bar{\nu}(\mathrm{E})=\inf \sum_{k} \rho\left(\left(A_{k}\right)_{t_{k}}\right)$, where the infimum is taken over the family $S$ of all finite and countable covers of E. It is well known that $\bar{\nu}$ is an outer measure. Now, we are going to show that the set $A_{t_{0}} \in \Sigma$ is $\bar{\nu}$-measurable for any fixed $A \in \mathcal{L}_{2 n}$ and $t_{0} \in(a, b)$. We want to prove that

$$
\bar{\nu}\left(A_{t_{0}} \cap E\right)+\bar{\nu}\left(A_{t_{0}}^{\prime} \cap E\right) \leq \bar{\nu}(E)
$$

for every set $E \subset \Omega$ and for $A_{t_{0}}^{\prime}=\Omega \backslash A_{t_{0}}$. If $\bar{\nu}(E)=\infty$ then our inequality is satisfied. 
Suppose that $\bar{\nu}(E)<\infty, E \subset \bigcup_{k}\left(A_{k}\right)_{t_{k}} \cup(\Omega \backslash \bar{C})$ and $\sum_{k} \rho\left(\left(A_{k}\right)_{t_{k}}\right)<\infty$, where $\left(A_{k}\right)_{t_{k}} \in \mathrm{D}$ for $k \in \mathbb{N}, t_{k} \in(a, b)$ and $A_{k} \in \mathcal{L}_{2 n}$. Let $\left(A_{k}\right)_{t_{k}}=$ $\left(B_{k}\right)_{t_{k}} \cup\left(D_{k}\right)_{t_{k}}$ for a fixed $k \in \mathbb{N}$ and $B_{k}, D_{k} \in \mathcal{L}_{2 n},\left(B_{k}\right)_{t_{k}}=A_{t_{0}} \cap\left(A_{k}\right)_{t_{k}}$ and $\left(D_{k}\right)_{t_{k}}=A_{t_{0}}^{\prime} \cap\left(A_{k}\right)_{t_{k}}$.

First we show that such sets $B_{k}, D_{k} \in \mathcal{L}_{2 n}$ exist. Let $U_{k}$ be an open set in $\mathbb{R}^{2 n}$ such that $\left(\mathbf{x}_{0}, \mathbf{y}_{0}\right) \in U_{k}$ if $t_{k} \in J$ for $\widetilde{\omega}_{\mathbf{x}_{0}, \mathbf{y}_{0}, t_{0}} \in \mathrm{C}$ and $\widetilde{\omega}_{\mathbf{x}_{0}, \mathbf{y}_{0}, t_{0}}$ : $J \rightarrow \mathbb{R}^{2 n}$. Let $\varphi_{k}: U_{k} \rightarrow \mathbb{R}^{2 n}$ be given by $\varphi_{k}\left(\mathbf{x}_{0}, \mathbf{y}_{0}\right)=\widetilde{\omega}_{\mathbf{x}_{0}, \mathbf{y}_{0}, t_{0}}\left(t_{k}\right)$ for $\left(\mathbf{x}_{0}, \mathbf{y}_{0}\right) \in U_{k}$. Under our assumptions about the system (Id), the function $\varphi_{k}$ locally satisfies the Lipschitz condition. So, $\varphi_{k}\left(A \cap U_{k}\right) \in \mathcal{L}_{2 n}$. Let $B_{k}=$ $\varphi_{k}\left(A \cap U_{k}\right) \cap A_{k}$. Hence, $B_{k} \in \mathcal{L}_{2 n}$ and $\left(B_{k}\right)_{t_{k}}=A_{t_{0}} \cap\left(A_{k}\right)_{t_{k}} \in \mathrm{D}$. Moreover, $D_{k} \in \mathcal{L}_{2 n}$ and $\left(D_{k}\right)_{t_{k}}=A_{t_{0}}^{\prime} \cap\left(A_{k}\right)_{t_{k}} \in \mathrm{D}$ for $D_{k}=A_{k} \backslash B_{k}$.

Now for $k \in \mathbb{N}$, we have $\rho\left(\left(A_{k}\right)_{t_{k}}\right)=\rho\left(\left(B_{k}\right)_{t_{k}}\right)+\rho\left(\left(D_{k}\right)_{t_{k}}\right), A_{t_{0}} \cap E \subset$ $\bigcup_{k}\left(B_{k}\right)_{t_{k}} \cup(\Omega \backslash \bar{C})$ and $A_{t_{0}}^{\prime} \cap E \subset \bigcup_{k}\left(D_{k}\right)_{t_{k}} \cup(\Omega \backslash \bar{C})$. Thus, we obtain

$$
\bar{\nu}\left(A_{t_{0}} \cap E\right)+\bar{\nu}\left(A_{t_{0}}^{\prime} \cap E\right) \leq \sum_{k} \rho\left(\left(B_{k}\right)_{t_{k}}\right)+\sum_{k} \rho\left(\left(D_{k}\right)_{t_{k}}\right)=\sum_{k} \rho\left(\left(A_{k}\right)_{t_{k}}\right)
$$

and hence

$$
\bar{\nu}\left(A_{t_{0}} \cap E\right)+\bar{\nu}\left(A_{t_{0}}^{\prime} \cap E\right) \leq \bar{\nu}(E) .
$$

So, the set $A_{t_{0}}$ is measurable with respect to $\bar{\nu}$.

Let $\nu$ be the measure on the $\sigma$-field $\Sigma$ such that $\nu(A)=\bar{\nu}(A)$ for all $A \in \Sigma$. The next step is to show that

$$
\nu\left(A_{t}\right)=\int_{A} d(\mathbf{x}, \mathbf{y}, t) d \mu(\mathbf{x}, \mathbf{y}) \quad \text { for } t \in(a, b) \text { and } A \in \mathcal{L}_{2 n} .
$$

Note that $(A)_{t_{0}} \in \mathrm{D}$ and $\rho\left((A)_{t_{0}}\right)=\int_{A} d\left(\mathbf{x}, \mathbf{y}, t_{0}\right) d \mu(\mathbf{x}, \mathbf{y})$ for fixed $t_{0} \in(a, b)$ and $A \in \mathcal{L}_{2 n}$. Thus, $\nu\left(A_{t_{0}}\right) \leq \int_{A} d\left(\mathbf{x}, \mathbf{y}, t_{0}\right) d \mu(\mathbf{x}, \mathbf{y})$. Let sets $\left(A_{k}\right)_{t_{k}} \in \mathrm{D}$, for $A_{k} \in \mathcal{L}_{2 n}, k \in \mathbb{N}$ and $t_{k} \in(a, b)$, be such that $A_{t} \subset \bigcup_{k}\left(A_{k}\right)_{t_{k}} \cup(\Omega \backslash \bar{C})$. Reasoning as previously, we obtain $A_{t_{0}} \cap\left(A_{k}\right)_{t_{k}}=\left(B_{k}\right)_{t_{k}} \in \mathrm{D}$ for a set $B_{k} \subset A_{k}$ with $B_{k} \in \mathcal{L}_{2 n}$. Let $V_{k}$ be an open set in $\mathbb{R}^{2 n}$ such that $\left(\mathbf{x}_{0}, \mathbf{y}_{0}\right) \in V_{k}$ if $t_{0} \in J$ for $\widetilde{\omega}_{\mathbf{x}_{0}, \mathbf{y}_{0}, t_{k}} \in \mathrm{C}$ and $\widetilde{\omega}_{\mathbf{x}_{0}, \mathbf{y}_{0}, t_{k}}: J \rightarrow \mathbb{R}$. Define $\varphi_{1, k}: V_{k} \rightarrow \mathbb{R}^{2 n}$ by $\varphi_{1, k}\left(\mathbf{x}_{0}, \mathbf{y}_{0}\right)=\widetilde{\omega}_{\mathbf{x}_{0}, \mathbf{y}_{0}, t_{k}}\left(t_{0}\right)$ for $\left(\mathbf{x}_{0}, \mathbf{y}_{0}\right) \in V_{k}, k \in \mathbb{N}$ and $\widetilde{\omega}_{\mathbf{x}_{0}, \mathbf{y}_{0}, t_{k}} \in \mathrm{C}$. The functions $\varphi_{1, k}, k \in \mathbb{N}$, locally satisfy the Lipschitz condition, so $\varphi_{1, k}\left(B_{k}\right) \in \mathcal{L}_{2 n}$ for $k \in \mathbb{N}$. Moreover,

$$
\int_{\varphi_{1, k}\left(B_{k}\right)} d\left(\mathbf{x}, \mathbf{y}, t_{0}\right) d \mu(\mathbf{x}, \mathbf{y})=\int_{B_{k}} d\left(\mathbf{x}, \mathbf{y}, t_{k}\right) d \mu(\mathbf{x}, \mathbf{y})
$$

because $d$ is a local integral invariant of the system (Id). Notice that $A_{t_{0}} \subset$ $\bigcup_{k}\left(B_{k}\right)_{t_{k}} \cup(\Omega \backslash \bar{C})$ and $\left(B_{k}\right)_{t_{k}}=\left(\varphi_{1, k}\left(B_{k}\right)\right)_{t_{0}}$ for $k \in \mathbb{N}$. So, $A_{t_{0}} \subset$ $\bigcup_{k}\left(\varphi_{1, k}\left(B_{k}\right)\right)_{t_{0}} \cup(\Omega \backslash \bar{C})$ and $A \subset \bigcup_{k} \varphi_{1, k}\left(B_{k}\right)$. Thus 


$$
\begin{aligned}
\int_{A} d\left(\mathbf{x}, \mathbf{y}, t_{0}\right) d \mu(\mathbf{x}, \mathbf{y}) \leq \sum_{k} \int_{\varphi_{1, k}\left(B_{k}\right)} d\left(\mathbf{x}, \mathbf{y}, t_{0}\right) d \mu(\mathbf{x}, \mathbf{y}) \\
=\sum_{k} \int_{B_{k}} d\left(\mathbf{x}, \mathbf{y}, t_{k}\right) d \mu(\mathbf{x}, \mathbf{y}) \leq \sum_{k} \int_{A_{k}} d\left(\mathbf{x}, \mathbf{y}, t_{k}\right) d \mu(\mathbf{x}, \mathbf{y}) \\
=\sum_{k} \rho\left(\left(A_{k}\right)_{t_{k}}\right)
\end{aligned}
$$

and hence $\int_{A} d\left(\mathbf{x}, \mathbf{y}, t_{0}\right) \leq \nu\left(A_{t_{0}}\right)$. So, $\nu\left(A_{t}\right)=\int_{A} d(\mathbf{x}, \mathbf{y}, t) d \mu(\mathbf{x}, \mathbf{y})$ for $A \in \mathcal{L}_{2 n}$ and $t \in(a, b)$. Hence, $\varrho_{X_{t}, Y_{t}}(\mathbf{x}, \mathbf{y})=d(\mathbf{x}, \mathbf{y}, t)$ for $t \in(a, b)$ and $\mathbf{x}, \mathbf{y} \in \mathbb{R}^{n}$. For the measure $\nu$ we have $\varrho_{X_{t}, Y_{t}}(\mathbf{x}, \mathbf{y})=d(\mathbf{x}, \mathbf{y}, t)=$ $p(\mathbf{y}, \mathbf{x}, t) c(\mathbf{x}, t), \varrho_{X_{t}}(\mathbf{x})=c(\mathbf{x}, t)$ and $w(\mathbf{x}, t, \Delta t)=\int_{\mathbb{R}^{n}} d(\mathbf{x}-\Delta t \mathbf{y}, \mathbf{y}, t) d \mu(\mathbf{y})$, for $\mathbf{x}, \mathbf{y} \in \mathbb{R}^{n}$ and $t, t+\Delta t \in(a, b)$. Therefore it follows from conditions $(1 \mathrm{~b})-(5 \mathrm{~b})$ and $(1 \mathrm{c})-(4 \mathrm{c})$ that the measure $\nu$ fulfills conditions $(\mathrm{a})-(\mathrm{d})$ in the definition of the family M. Moreover, by our assumptions equation (Ic) is equivalent to condition (4a), so $\nu \in \mathrm{M}$.

The measure $\nu$ given in the proof of Theorem 2 is not unique. So, the conditional differential equation does not have a unique solution. However, using Theorems 11 and 2 we can uniquely determine the functions $\varrho_{X_{t}}$ for $t \in(a, b)$. In some situations this may be sufficient: see the example in the next section. It should be emphasized that conditional differential equations describe much more general situations than is described by the system (Id) in the proof of Theorem 2 .

Let us consider the space $\bar{\Omega} \subset \Omega$ such that

$$
\begin{aligned}
\bar{\Omega}=\left\{\omega \in \Omega: \omega:(a, b) \rightarrow \mathbb{R}^{n}, \lim _{t \rightarrow a^{+}}\left\|\left(\omega(t), \omega^{\prime}(t)\right)\right\|_{\mathbb{R}^{2 n}} \neq \infty,\right. \\
\\
\left.\lim _{t \rightarrow b^{-}}\left\|\left(\omega(t), \omega^{\prime}(t)\right)\right\|_{\mathbb{R}^{2 n}} \neq \infty\right\} .
\end{aligned}
$$

We endow $\bar{\Omega}$ with the metric $\rho$ given by $\rho\left(\omega_{1}, \omega_{2}\right)=\sup _{t \in(a, b)} \|\left(\omega_{1}(t)-\omega_{2}(t)\right.$, $\left.\omega_{1}^{\prime}(t)-\omega_{2}^{\prime}(t)\right) \|_{\mathbb{R}^{2 n}}$ for $\omega_{1}, \omega_{2} \in \bar{\Omega}$. Let $\Gamma$ be the $\sigma$-field on $\bar{\Omega}$ defined just as in $\Omega$. Notice that $\Gamma \subset \Sigma$. Basing on the proof of Theorem 2, we can construct a measure $\nu \in \mathrm{M}$ such that

(a) $\nu$ is a solution of the conditional differential equation from Theorem 2 ,

(b) its support is the whole space $\bar{\Omega}$ if the measure $\nu$ is restricted to the space $(\bar{\Omega}, \Gamma)$.

Assumptions (1a)-(3a) in the definition of conditional differential equations are fulfilled by most of distributions which are used in applications.

Notice that condition (4a) is very general. So, we are able to describe a lot of real phenomena using equation (Ic).

REMARK 1. The verification of assumption (5a) in Theorem 2 can be difficult in applications. Notice that the function $c=c(\mathbf{x}, t)$, for $\mathbf{x} \in \mathbb{R}^{n}$ and 
$t \in(a, b)$, is a local integral invariant of the system of differential equations

$$
x_{1}^{\prime}=E_{1}(\mathbf{x}, t), \ldots, x_{n}^{\prime}=E_{n}(\mathbf{x}, t)
$$

for $\mathbf{x} \in \mathbb{R}^{n}$ and $t \in(a, b)$ (cf. [3, Theorem 2]). Suppose that

(6b) the functions $E_{i}=E_{i}(\mathbf{x}, t)$ fulfill the Lipschitz condition with respect to $\mathbf{x}$.

Then solutions of (IId) are uniquely determined on $(a, b)$. Thus, condition (5a) in Theorem 2 results from condition (6b) and condition (2c) in Theorem 2 (cf. [3, Theorem 2]).

Assumption (4a) is in fact equivalent to equation (Ic) (see the proof of Theorem 1). We can use (Ic) to determine the probability density functions for the random variables $X_{t}, t \in(a, b)$. Recall that $\varrho_{X_{t}}(\mathbf{x})=c(\mathbf{x}, t)$ for $\mathbf{x} \in \mathbb{R}^{n}$ and $t \in(a, b)$. Naturally condition (4a) is not always true in applications. We can assume that equation (Ic) is realistic in the case of phenomena for which trajectories $\omega \in \Omega$ with large values $\left\|\omega^{\prime \prime}(t)\right\|_{\mathbb{R}^{n}}$ for $t \in(a, b)$ are rare. The following theorem explains this.

TheOREm 3. Under the notation of Section 3, assume that

(a) the measure $\nu$ is defined on the $\sigma$-field $\Sigma$,

(b) the functions $c=c(\mathbf{x}, t), p=p(\mathbf{y}, \mathbf{x}, t)$ and $E_{i}=E_{i}(\mathbf{x}, t)$, for $\mathbf{x}, \mathbf{y} \in \mathbb{R}^{n}$ and $t \in(a, b)$, are determined by $\nu$ as in the definition of the family $\mathrm{M}$ in Section 3,

(c) the functions $c, p$ and $E_{i}, i=1, \ldots, n$, satisfy conditions (1a)-(3a) and $(1 \mathrm{~b})-(5 \mathrm{~b})$.

For $r>0$, set

$$
H_{r}=\left\{\omega \in \Omega: \text { there exists } t \in(a, b) \text { such that }\left\|\omega^{\prime \prime}(t)\right\|_{\mathbb{R}^{n}}>r\right\} .
$$

Suppose that

(5ax) for the outer measure $\bar{\nu}$ induced by $\nu$ there exists $r_{0}>0$ such that $\bar{\nu}\left(H_{r}\right) \leq 1 / r^{2}$ for $r>r_{0}$.

Then the measure $\nu$ satisfies condition (4a) and $\nu \in \mathrm{M}$.

Proof. We keep the notation of Sections 2 and 3. Let $t \in(a, b)$ and $\Delta t \in \mathbb{R}$ with $t+\Delta t \in(a, b)$. Fix a cuboid $K \subset \mathbb{R}^{n}, K=\left[a_{1}, b_{1}\right] \times \cdots \times\left[a_{n}, b_{n}\right]$, and $r>0$. Set

$$
K_{-}^{+} r(\Delta t)^{2}=\left[a_{1}-r(\Delta t)^{2}, b_{1}+r(\Delta t)^{2}\right] \times \cdots \times\left[a_{n}-r(\Delta t)^{2}, b_{n}+r(\Delta t)^{2}\right] .
$$

Define

$$
\begin{aligned}
A & =\left\{\omega \in \Omega: W_{t, \Delta t}(\omega) \in K\right\}, \\
B_{r, \Delta t} & =\left\{\omega \in \Omega: \omega(t+\Delta t) \in K_{-}^{+} r(\Delta t)^{2}\right\} .
\end{aligned}
$$


Notice that $A \in \Sigma$ and $B_{r, \Delta t} \in \Sigma$. Taking into account the Taylor formula for $\omega \in \Omega$ and the definitions of $A, B_{r, \Delta t}, H_{r}$, we obtain $A \subset B_{r, \Delta t} \cup H_{r}$. Thus

$$
\nu(A) \leq \bar{\nu}\left(B_{r, \Delta t} \cup H_{r}\right) \leq \nu\left(B_{r, \Delta t}\right)+\bar{\nu}\left(H_{r}\right) .
$$

Therefore

$$
\int_{K} w(\mathbf{x}, t, \Delta t) d \mu(\mathbf{x}) \leq \int_{K_{-}^{+} r(\Delta t)^{2}} c(\mathbf{x}, t+\Delta t) d \mu(\mathbf{x})+\bar{\nu}\left(H_{r}\right) .
$$

From the above inequalities and assumption (3a) about $c$, we obtain

$$
\begin{aligned}
\int_{K}(w(\mathbf{x}, t, \Delta t)-c(\mathbf{x}, t+\Delta t)) d \mu(\mathbf{x}) \\
\leq \int_{\left(K_{-}^{+} r(\Delta t)^{2}\right) \backslash K} c(\mathbf{x}, t+\Delta t) d \mu(\mathbf{x})+\bar{\nu}\left(H_{r}\right) \\
\leq a \mu\left(\left(K_{-}^{+} r(\Delta t)^{2}\right) \backslash K\right)+\bar{\nu}\left(H_{r}\right) \\
\leq a s r(\Delta t)^{2}+\bar{\nu}\left(H_{r}\right),
\end{aligned}
$$

where $s$ is a constant coefficient depending on $K$. We can assume that $s$ is independent of $r$ and $\Delta t$ for $\Delta t$ sufficiently small and $r<1 /|\Delta t|$. Let

$$
\begin{aligned}
C & =\{\omega \in \Omega: \omega(t+\Delta t) \in K\}, \\
D_{r, \Delta t} & =\left\{\omega \in \Omega: W_{t, \Delta t}(\omega) \in K_{-}^{+} r(\Delta t)^{2}\right\} .
\end{aligned}
$$

We have $C \in \Sigma, D_{r, \Delta t} \in \Sigma$ and $C \subset D_{r, \Delta t} \cup H_{r}$. By the formula for $w$ and assumptions (3a) and (4b), for sufficiently small $\Delta t$, we have $w(\mathbf{x}, t, \Delta t)<b$ for all $\mathbf{x} \in K_{-}^{+} r(\Delta t)^{2}$ and $r<1 /|\Delta t|$, with some $b>0$. Therefore

$$
\nu(C) \leq \bar{\nu}\left(D_{r, \Delta t} \cup H_{r}\right) \leq \nu\left(D_{r, \Delta t}\right)+\bar{\nu}\left(H_{r}\right)
$$

and

$$
\int_{K} c(\mathbf{x}, t+\Delta t) d \mu(\mathbf{x}) \leq \int_{K_{-}^{+} r(\Delta t)^{2}} w(\mathbf{x}, t, \Delta t) d \mu(\mathbf{x})+\bar{\nu}\left(H_{r}\right) .
$$

Hence

$$
\begin{aligned}
\int_{K}(c(\mathbf{x}, t+\Delta t)-w(\mathbf{x}, t, \Delta t)) d \mu(\mathbf{x}) & \leq \int_{\left(K_{-r}^{+} r(\Delta t)^{2}\right) \backslash K} w(\mathbf{x}, t, \Delta t) d \mu(\mathbf{x})+\bar{\nu}\left(H_{r}\right) \\
& \leq b \mu\left(\left(K_{-}^{+} r(\Delta t)^{2}\right) \backslash K\right)+\bar{\nu}\left(H_{r}\right) \\
& \leq b r s(\Delta t)^{2}+\bar{\nu}\left(H_{r}\right),
\end{aligned}
$$

where $s$ is as previously. So,

$$
\begin{aligned}
-\left(\operatorname{ars} \Delta t+\frac{\bar{\nu}\left(H_{r}\right)}{\Delta t}\right) & \leq \int_{K} \frac{c(\mathbf{x}, t+\Delta t)-w(\mathbf{x}, t, \Delta t)}{\Delta t} d \mu(\mathbf{x}) \\
& \leq b r s \Delta t+\frac{\bar{\nu}\left(H_{r}\right)}{\Delta t}
\end{aligned}
$$

for $\Delta t$ sufficiently small and $r<1 /|\Delta t|$. Substituting $r=1 /|\Delta t|^{\alpha}(1 / 2<$ $\alpha<1$ and $|\Delta t|<1)$ in the above inequalities and taking into account 
condition (5ax), we obtain

$$
\lim _{\Delta t \rightarrow 0} \int_{K} \frac{c(\mathbf{x}, t+\Delta t)-w(\mathbf{x}, t, \Delta t)}{\Delta t} d \mu(\mathbf{x})=0
$$

for each fixed cuboid $K \subset \mathbb{R}^{n}$. Under our assumptions,

$$
\begin{aligned}
\frac{c(\mathbf{x}, t+\Delta t)-w(\mathbf{x}, t, \Delta t)}{\Delta t} & \frac{\partial c}{\partial t}(\mathbf{x}, t)+\sum_{i=1}^{n} \frac{\partial\left(c E_{i}\right)}{\partial x_{i}}(\mathbf{x}, t)+\left(\frac{\partial c}{\partial t}\left(\mathbf{x}, t+\theta_{1, \mathbf{x}}\right)-\frac{\partial c}{\partial t}(\mathbf{x}, t)\right) \\
& +\sum_{i=1}^{n} \int_{\mathbb{R}^{n}} y_{i}\left(\frac{\partial d}{\partial x_{i}}\left(\mathbf{x}-\theta_{2, \mathbf{y}} \mathbf{y}, \mathbf{y}, t\right)-\frac{\partial d}{\partial x_{i}}(\mathbf{x}, \mathbf{y}, t)\right) d \mu(\mathbf{y}),
\end{aligned}
$$

where $\theta_{1, \mathbf{x}}, \theta_{2, \mathbf{y}}$ are between 0 and $\Delta t$ (see the proof Theorem 1). Thus, for sufficiently small $\Delta t$, for $\mathbf{x} \in K$ and fixed $t \in(a, b)$, the values of $\frac{c(\mathbf{x}, t+\Delta t)-w(\mathbf{x}, t, \Delta t)}{\Delta t}$ are uniformly bounded (conditions (3a), (4b)). So, we have

$$
\begin{aligned}
\lim _{\Delta t \rightarrow 0} \int_{K} \frac{c(\mathbf{x}, t+\Delta t)-w(\mathbf{x}, t, \Delta t)}{\Delta t} & d \mu(\mathbf{x}) \\
& =\int_{K} \lim _{\Delta t \rightarrow 0} \frac{c(\mathbf{x}, t+\Delta t)-w(\mathbf{x}, t, \Delta t)}{\Delta t} d \mu(\mathbf{x}) .
\end{aligned}
$$

Thus

$$
\int_{K} \lim _{\Delta t \rightarrow 0} \frac{c(\mathbf{x}, t+\Delta t)-w(\mathbf{x}, t, \Delta t)}{\Delta t} d \mu(\mathbf{x})=0
$$

for each fixed cuboid $K$ and each $t \in(a, b)$. Let

$$
h(\mathbf{x}, t)=\lim _{\Delta t \rightarrow 0} \frac{c(\mathbf{x}, t+\Delta t)-w(\mathbf{x}, t, \Delta t)}{\Delta t} .
$$

We have $h(\mathbf{x}, t)=\frac{\partial c}{\partial t}(\mathbf{x}, t)+\sum_{i=1}^{n} \frac{\partial\left(c E_{i}\right)}{\partial x_{i}}(\mathbf{x}, t)$ (see the proof of Theorem 1). Notice that $h=h(\mathbf{x}, t)$ is a continuous function of $\mathbf{x} \in \mathbb{R}^{n}$ for each fixed $t \in(a, b)$. It follows that

$$
\lim _{\Delta t \rightarrow 0} \frac{c(\mathbf{x}, t+\Delta t)-w(\mathbf{x}, t, \Delta t)}{\Delta t}=0
$$

for each $\mathbf{x} \in \mathbb{R}^{n}$ and $t \in(a, b)$.

Condition (5ax) means that $P_{t_{0}}(A)=\nu(A)<1 / r^{2}$ for each fixed $t_{0} \in$ $(a, b)$ and for all events $A \in \Sigma_{t_{0}}$ included in $H_{r}$. For specific mathematical models, events $A \in \Sigma_{t_{0}}, A \subset H_{r}$, can have some interpretation. So, condition (5ax) is intuitively more clear than (4a). However, (4a) is much more general. 
5. Random model of Malthus type for the population size. We will use the notation and definitions of Section 3 for $n=1$.

Assume that various populations of a species $K$ can live in a region $P$. We will consider a random model of population size of such populations.

Fix an interval $(a, b) \subset \mathbb{R}$ such that $0 \in(a, b)$. Suppose that some population of species $K$ stays in region $P$ in the time interval $J \subset(a, b)$. Let $\omega: J \rightarrow \mathbb{R}$ describe the dependence of the number of individuals of this population on time. We assume that $\omega$ can be any element of the set $\Omega$. Suppose that populations in our model can migrate from and into $P$. We assume that such migrations can occur when sudden changes of the population size appear. So, in our model, functions $\omega \in \Omega$ for which $\lim _{t \rightarrow \alpha^{+}}\left\|\left(\omega(t), \omega^{\prime}(t)\right)\right\|_{\mathbb{R}^{2}}$ $=\infty$ and $\lim _{t \rightarrow \beta^{-}}\left\|\left(\omega(t), \omega^{\prime}(t)\right)\right\|_{\mathbb{R}^{2}}=\infty$ for $\alpha, \beta \in[a, b]$ take into account migrations of observed populations.

Let us take a measure $\nu \in \mathrm{M}$ defined on the space $(\Omega, \Sigma)$. For each fixed $t \in(a, b)$ we consider the probability $P_{t}$ on $\left(\Omega_{t}, \Sigma_{t}\right)$ given by $P_{t}(\mathrm{~A})=\nu(\mathrm{A})$ for $\mathrm{A} \in \Sigma_{t}$. This probability measures the chance that a population of size described by a function $\omega \in \mathrm{A}, \mathrm{A} \in \Sigma_{t}$, appears in region $P$. For a fixed $t \in(a, b)$, the function $c=c(\cdot, t)$ is the probability density function for the random variable $X_{t}$. Recall that for $\omega \in \Omega_{t}, t \in(a, b)$, we have $X_{t}(\omega)=\omega(t)$ and $\omega(t)$ is the population size at time $t$. Also recall that $Y_{t}(\omega)=\omega^{\prime}(t)$ for $\omega \in \Omega_{t}$. We will write $f(x) \sim N(m, \sigma)$ whenever $f(x)$ is the probability density function of the normal distribution with expected value $m \in \mathbb{R}$ and standard deviation $\sigma>0$.

To prepare our model we assume that we look for a measure $\nu \in \mathrm{M}$ for which

$$
f_{Y_{t}}\left(y \mid X_{t}=x\right)=\frac{1}{\sqrt{2 \pi} \sigma_{1}} \exp \left(-\frac{(y-k x)^{2}}{2 \sigma_{1}^{2}}\right) \sim N\left(k x, \sigma_{1}\right),
$$

where $k \in \mathbb{R}$. Moreover, we suppose that

$$
c(x, 0)=\frac{1}{\sqrt{2 \pi} \sigma} \exp \left(-\frac{\left(x-m_{0}\right)^{2}}{2 \sigma^{2}}\right) \sim N\left(m_{0}, \sigma\right),
$$

where $m_{0}>0$.

We consider such a model because it is known that the random values of the population size of many populations and their increases have normal distributions. Moreover, we take into account the classical Malthus model of growth described by the differential equation $x^{\prime}=k x$ for $k \in \mathbb{R}$ and the initial condition $x(0)=m_{0}, m_{0}>0(x:(a, b) \rightarrow \mathbb{R})$. Condition (Ia) takes into account that the most probable development of the population at time $t \in(a, b)$ is described by the relationship $x^{\prime}=k x$. Thus, on the space $(\Omega, \Sigma)$, we consider the conditional differential equation (Ia) with the initial 
condition $(\mathrm{Ib})$. It is easy to see that the function given by

$$
p(y, x, t)=\frac{1}{\sqrt{2 \pi} \sigma_{1}} \exp \left(-\frac{(y-k x)^{2}}{2 \sigma_{1}^{2}}\right) \sim N\left(k x, \sigma_{1}\right)
$$

for $x, y \in \mathbb{R}$ and $t \in(a, b)$, satisfies conditions (1b)-(4b), and the function $\rho_{0}: \mathbb{R} \rightarrow \mathbb{R}$ given by $\rho_{0}(x) \sim N\left(m_{0}, \sigma\right)$ for $x \in \mathbb{R}$ satisfies conditions $(1 \mathrm{c})-(4 \mathrm{c})$. Moreover, $E(x, t)=\int_{\mathbb{R}} y p(y, x, t) d \mu(y)=k x$ for $x, y \in \mathbb{R}$ and $t \in(a, b)$. Notice that the function $E=E(x, t)$ for $x \in \mathbb{R}$ and $t \in(a, b)$ satisfies conditions $(5 \mathrm{~b}),(6 \mathrm{~b})$. Thus, our considerations lead to the equation

$$
\frac{\partial c}{\partial t}+\frac{\partial(k x c)}{\partial x}=0 \quad \text { with } c(x, 0) \sim N\left(m_{0}, \sigma\right) \text { for } x \in \mathbb{R}
$$

(cf. Theorem 1). The function $c: \mathbb{R} \times(a, b) \rightarrow \mathbb{R}$ such that

$$
c(x, t)=\frac{1}{\sigma e^{k t} \sqrt{2 \pi}} \exp \left(-\frac{\left(x-m_{0} e^{k t}\right)^{2}}{2\left(\sigma e^{k t}\right)^{2}}\right)
$$

for $x \in \mathbb{R}$ and $t \in(a, b)$ is a solution of (Ic). The functions $c, p$ and $\rho_{0}$ fulfill the assumptions of Theorem 2 . Thus, the conditional differential equation (Ia) with the initial condition (Ib) has a solution $\nu \in \mathrm{M}$ (Theorem 2). Moreover, for each such solution,

$$
\varrho_{X_{t}}(x)=c(x, t)=\frac{1}{\sigma e^{k t} \sqrt{2 \pi}} \exp \left(-\frac{\left(x-m_{0} e^{k t}\right)^{2}}{2\left(\sigma e^{k t}\right)^{2}}\right)
$$

for $x \in \mathbb{R}$ and $t \in(a, b)$ (Theorem 1 ).

From our model we obtain the following conclusions:

Conclusion 1. In our model, for fixed $t \in(a, b)$, the random values of the population size have the normal distribution $N(m(t), \sigma(t))$ with $m(t)=$ $m_{0} e^{k t}$ and $\sigma(t)=\sigma e^{k t}$.

Conclusion 2. Let us consider the expected value of the population size in our model as a function of time, $m:(a, b) \rightarrow \mathbb{R}$. Then $m$ is the solution of the differential equation $x^{\prime}=a x$ with the initial condition $x(0)=m_{0}$ considered in the Malthus model. So, if $\sigma_{1}, \sigma \approx 0$ our random model reduces to the classical Malthus model.

In our model, values of functions $\omega \in \Omega$ may be negative. This is a defect of the model, as such values have no interpretation as population size. However in our model, $c(x, t) \approx 0$ for each fixed $t$ and $x<0$. So, we can still regard our model as good.

Our results are consistent with intuition and, under some conditions, with experimental data. The above model shows that we can get results credibly describing reality using conditional differential equations. Moreover, conditional differential equations give other possibilities of applications than those available with the use of standard stochastic equations. 


\section{References}

[1] J. Banasiak, A. Goswami and S. Shindin, Singularly perturbed population models with reducible migration matrix: 2. Asymptotic analysis and numerical simulations, Mediterr. J. Math. 11 (2014), 533-559.

[2] L. Kenneth and J. L. Kuttler, Generalized stochastic evolution equations, J. Differential Equations 257 (2014), 816-842.

[3] C. Rom, A version of non-hamiltonian Liouville equation, Discuss. Math. Differ. Incl. Control Optim. 34 (2014), 5-14.

[4] K. Sobczyk, Stochastic Differential Equations: with Applications to Physics and Engineering, Springer, 2001.

Celina Rom

Department of Mathematics

University of Bielsko-Biała

43-309 Bielsko-Biała, Poland

E-mail: crom@ath.bielsko.pl 
